\title{
Erratum to: Gravitational geometric phase in the presence of torsion
}

\section{Knut Bakke $^{\mathrm{a}}$, Claudio Furtado ${ }^{\mathrm{b}}$, J.R. Nascimento}

Departamento de Física, Universidade Federal da Paraíba, Caixa Postal 5008, 58051-970 João Pessoa, PB, Brazil

Received: 20 July 2009 / Published online: 19 September 2009

(C) Springer-Verlag / Società Italiana di Fisica 2009

Erratum to: Eur. Phys. J. C (2009) 60: 501-507 DOI 10.1140/epjc/s10052-009-0944-z

List of corrections in the article [1].

1. Equation (4.9) should read

$$
\begin{aligned}
\phi_{T} & =\frac{1}{8} \oint e_{\varphi}^{i} S^{0} \Sigma_{i} \mathrm{~d} \varphi=\frac{1}{8} \oint S^{0} \vec{\Sigma} \cdot \overrightarrow{\mathrm{d} r} \\
& =\frac{1}{8} \oint S^{0} \Sigma^{\varphi} \eta \rho \mathrm{d} \varphi=\pi \eta \chi \Sigma^{2},
\end{aligned}
$$

where we had defined the matrix $\Sigma^{\varphi}=-\sin \varphi \Sigma^{1}+$ $\cos \varphi \Sigma^{2}$.

2. Equation (5.12) must be written in the form

$$
\vec{\Xi}=\mu \hat{\beta} \vec{\Sigma} \times \vec{E}-d \hat{\beta} \vec{\Sigma} \times \vec{B}-\mathrm{i} \vec{\xi}+\frac{1}{8} S_{0} \vec{\Sigma}
$$

3. Equation (6.4) should read

$$
\begin{aligned}
\Phi & =\oint e_{\mu}^{i}\left(-\mathrm{i} \xi_{i}\right) \mathrm{d} x^{\mu}-\frac{1}{8} \oint S^{0} \sigma^{\varphi} \eta \rho \mathrm{d} \varphi \\
& =(1-\eta) \pi \sigma^{3}-\pi \eta \chi \sigma^{2},
\end{aligned}
$$

where $\sigma^{\varphi}=-\sin \varphi \sigma^{1}+\cos \varphi \sigma^{2}$.

4. Due to the change in (6.4) the term $\frac{\pi}{2} \chi \sigma^{3}$ in the phases (6.5), (6.6) and (6.7) changes to $-\pi \eta \chi \sigma^{2}$.

We claim that none these corrections affects the physical conclusion of the paper [1].

\section{References}

1. K. Bakke, C. Furtado, J.R. Nascimento, Eur. J. Phys. C 60, 501 (2009)

The online version of the original article can be found under doi:10.1140/epjc/s10052-009-0944-z.

a e-mail: kbakke@ fisica.ufpb.br

b e-mail: furtado@fisica.ufpb.br 